\title{
Dielectric properties under high electric field for silicon doped alumina thin film with glass-like structure derived from sol-gel
}

\author{
process

\begin{abstract}
Manwen Yao ${ }^{*}$, Zhen $\mathrm{Su}^{\dagger}$, Pei Zou, Jianwen Chen, Fei Li, Qian Feng, Xi Yao
\end{abstract} \\ Functional Materials Research Laboratory, School of Materials Science \& Engineering, Tongji \\ University, No. 4800 Caoan Road, Shanghai 201804, China
}

\begin{abstract}
Dielectric $\mathrm{Al}_{2-\mathrm{x}} \mathrm{Si}_{\mathrm{x}} \mathrm{O}_{\mathrm{y}}(\mathrm{X}=0.00,0.02,0.05,0.10)$ thin films were deposited onto $\mathrm{Pt} / \mathrm{Ti} / \mathrm{SiO}_{2} / \mathrm{Si}$ substrates using sol-gel spin coating technology. The obtained materials were characterized via differential scanning calorimetry (DSC), scanning electron microscopy (SEM), fourier transform infrared spectrometry (FT-IR) and X-ray photoelectron spectrometry (XPS). The results show that the films are amorphous with $\mathrm{Si}$ atoms occupying $\mathrm{Al}$ atom sites forming Al-O-Si bonds and glass-like structure. The dielectric properties of the film were investigated. By means of silicon doping, the leakage current and the dielectric loss of the amorphous alumina films much reduced while the breakdown strength enhanced. Two orders of magnitude reduction in leakage current and significant enhancement in breakdown strength (up to $566 \mathrm{MV} / \mathrm{m}$ ) can be achieved. The improved dielectric properties are attributed to the forming of Al-O-Si bonds and cation vacancies by the Si-addition. The structure modification enhanced the stability of alumina structure and promoted the ionic transportation to repair the defects of the alumina films.
\end{abstract}

Keywords: Alumina thin films, Si doping, Glass-like structure, Breakdown strength, Leakage current, Dielectric constant and loss

\section{Introduction}

In the recent decade, the development of green energy technology promoted extensive research effort on energy storage technology. Dielectric capacitors as a kind traditional energy storage device are still highly important for high density energy

\footnotetext{
* Corresponding author. E-mail address: yaomw@ @ongji.edu.cn

${ }^{\dagger}$ These authors contributed equally to this work and should be considered co-first authors.
} 
storage applications [1-3]. For dielectric energy storage applications, high dielectric constant and high breakdown strength are highly desirable. Polymer and polymer composite with very high electric breakdown strength are widely used in capacitor industry to develop high voltage and high energy storage capacitors $[4,5]$. Inorganic ceramic and glass ceramic dielectrics are another alternative perspective way to develop high performance energy storage devices.

Alumina $\left(\mathrm{Al}_{2} \mathrm{O}_{3}\right)$, as an excellent insulating dielectric material, is widely used as substrate and packaging material in electronics and microelectronics [6]. Moreover, because of its high breakdown strength (300 700 MV/m), high relative permitivity (8.6 10) and high band gap ( $\sim 9 \mathrm{eV})$ in conjunction with its excellent chemical and thermal stability, more and more attentions has been drawn to explore its potential applications in high energy density storage capacitors [7].

Alumina thin film can be prepared via conventional sol-gel and spin coating route. Dense, homogeneous and uniform film can be prepared at low temperatures [8]. One of the distinct advantages of the sol-gel technology is: the chemical composition and phase composition of the material can be easily and precisely controlled. Recently, the substitution effect of doping elements $\left(\mathrm{Ti}^{4+}, \mathrm{La}^{3+}\right)$ on the leakage current and breakdown characteristic of amorphous alumina thin films has been studied and excellent dielectric properties of doped alumina thin films were achieved [9, 10]. Silicon is one of the very common elements in glasses and ceramics. Typically, Silicate glass has a high hardness and mechanical strength owing to the forming of a stable amorphous Si-O network structure [11, 12]. It is interesting to see if silicon can be introduced into the amorphous alumina thin film to enhance the stability and dielectric behavior of the alumina structure. The current work is mainly focused on the dielectric properties of Si-doped alumina thin film under high electric field.

\section{Experimental}

\subsection{Sample preparation}

The $\mathrm{Al}_{2-\mathrm{x}} \mathrm{Si}_{\mathrm{x}} \mathrm{O}_{\mathrm{Y}}(\mathrm{x}=0,0.02,0.05$ and 0.1$)$ thin films represented as $\mathrm{Si}-0 \%, \mathrm{Si}-2 \%$, 
$\mathrm{Si}-5 \%, \mathrm{Si}-10 \%$ respectively, were prepared by the sol-gel and spin coating technology. Aluminum isopropoxide $\left(\mathrm{Al}\left(\mathrm{OC}_{3} \mathrm{H}_{7}\right)_{3}\right)$ and tetraethyl orthosilioate $\left(\mathrm{C}_{8} \mathrm{H}_{20} \mathrm{O}_{2} \mathrm{Si}\right)$ were used as Al-precursor and Si dopant, respectively. Firstly, both aluminum isopropoxide and tetraethyl orthosilioate were dissolved in glycol ether under constant stirring at 60 ${ }^{\circ} \mathrm{C}$ for $30 \mathrm{~min}$. Secondly, $0.02 \mathrm{~mol}$ acetylacetone acting as chelating agent was added to restrain the hydrolysis of Aluminum isopropoxide with stirring for $30 \mathrm{~min}$. The mixture was then heated to $90{ }^{\circ} \mathrm{C}$ and $10 \mathrm{ml}$ acetic acid as catalyst was added to the above solution with agitation for $30 \mathrm{~min}$. Finally, the mixture was cooled down to room temperature to get a clear and homogeneous sol. Prior to spin coating deposition of the film, substrates $\left(\mathrm{Pt} / \mathrm{Ti} / \mathrm{SiO}_{2} / \mathrm{Si}\right)$ were ultrasonically rinsed in acetone, deionized water and ethyl alcohol, successively, and dried by blowing nitrogen $\mathrm{N}_{2}$.

Thin films were deposited on the substrate by spin-coating method at $3000 \mathrm{rpm}$ for $20 \mathrm{~s}$ per each layer in a clean-room environment. After each deposition, the films were preheated in a tubular furnace at $450{ }^{\circ} \mathrm{C}$ for $5 \mathrm{~min}$ to form solid films through evaporating the solvents and burning-out organic residuals. The above procedure was repeated for 7 times to obtain samples. Samples were annealed at $600{ }^{\circ} \mathrm{C}$ for $3 \mathrm{~h}$ with a heating speed of $3{ }^{\circ} \mathrm{C} / \mathrm{min}$ and then cooled down to room temperature in a muffle furnace. Gold was deposited on to the alumina film using a vacuum evaporation instrument (ZHD-400, Technol Science, China) via a mask as top electrode to form Metal-Insulator-Metal (MIM) structure with $\mathrm{Pt}$ as bottom electrode for the measurement of the dielectric properties. The diameter of the Au top electrode is 1 $\mathrm{mm}$.

\subsection{Characterizations}

Surface morphology of Si-doped alumina thin films was characterized by field emission scanning electron microscopy (FESEM) (S-4700, Hitachi, Japan). The thickness was measured by a dual beam laser interferometer (F20 Filmetrics Inc. San Diego, US). The existence form of silicon in Si-doped alumina thin films was analyzed by fourier transform infrared spectroscopy (FT-IR) (EQUINOX 55, Bruker Optics, Germany) and X-ray photoelectron spectroscopy (XPS) (ESCALAB 250Xi, 
Thermo Fisher, USA). The combustion temperature of organics and crystallization of the samples was recorded by differential scanning calorimeter (DSC) (SAT449C, Netzsch, Germany) from $30{ }^{\circ} \mathrm{C}$ to $1200{ }^{\circ} \mathrm{C}$ at a heating rate of $10{ }^{\circ} \mathrm{C} / \mathrm{min}$ in nitrogen atmosphere. Dielectric behaviors of the alumina film samples were measured. Before the measurements, samples were treated at $100^{\circ} \mathrm{C}$ for $30 \mathrm{~min}$ to remove any adsorbed water. The leakage current as a function of voltage was measured using a Keithley 2400 source meter unit interfaced with a computer to perform the measurements and record the experimental data. Voltage was applied in a successive voltage step of 0.2 V/step with a delay time of $0.1 \mathrm{~s} / \mathrm{step}$, until the leakage current increased sharply and abruptly, indicating the occurrence of breakdown. The positive voltage was applied to the Au top electrode, and the Pt bottom electrode was grounded. For each sample, 10 breakdown trials were tested to estimate the breakdown strength through Weibull distribution function due to the randomness of the dielectric breakdown voltage. The dielectric constant and loss of the alumina film were measured from $100 \mathrm{~Hz}$ to $2 \mathrm{MHz}$ using a LCR meter (E4980A, Agilent, USA).

\section{Results and discussion}

\subsection{DSC analysis of samples}

The thermal decomposition and transformation processes of the $\mathrm{Si}-0 \%, \mathrm{Si}-2 \%$, $\mathrm{Si}-5 \%$ and $\mathrm{Si}-10 \%$ samples at a heating rate of $10{ }^{\circ} \mathrm{C} / \mathrm{min}$ in nitrogen $(99.99 \%$ purity) atmosphere are described by the differential scanning calorimetry curves as shown in Fig. 1. The thermal decomposition and transformation processes of the samples can be divided into three different stages. During the initial stage (S-1), when the temperature is low $\left(30-320{ }^{\circ} \mathrm{C}\right)$, there are two endothermic peaks at around $85{ }^{\circ} \mathrm{C}$ and less than 300 ${ }^{\circ} \mathrm{C}\left(214{ }^{\circ} \mathrm{C}\right.$ for doped thin films and $296{ }^{\circ} \mathrm{C}$ for undoped thin film). The first peak is related to the evaporation of the physisorbed water, while the peak at higher temperature should be attributed to the loss of the chemisorbed water. As for the second stage (S-2), the temperature extends from $320{ }^{\circ} \mathrm{C}$ to $640{ }^{\circ} \mathrm{C}$. There is a broad exothermic peak during the entire temperature ramping course and it is ascribed to the thermal decomposition of organics of the film. For the third stage (S-3), when the 
temperature is high $\left(640{ }^{\circ} \mathrm{C}-1200{ }^{\circ} \mathrm{C}\right)$, all the samples present an endothermic peak except for the undoped Si-0\% sample, where two endothermic peaks can be found. The first peak is attributed to the transition from amorphous $\mathrm{Al}_{2} \mathrm{O}_{3}$ to the $\gamma-\mathrm{Al}_{2} \mathrm{O}_{3}$ at $876{ }^{\circ} \mathrm{C}, 924{ }^{\circ} \mathrm{C}, 973{ }^{\circ} \mathrm{C}$ and $989{ }^{\circ} \mathrm{C}$ respectively with the increasing of Si content. The second peak of the $\mathrm{Si}-0 \%$ at $1189{ }^{\circ} \mathrm{C}$ results from the transformation of the $\gamma-\mathrm{Al}_{2} \mathrm{O}_{3}$ to $\alpha-\mathrm{Al}_{2} \mathrm{O}_{3}$. Based on the above analysis, the ultimate thermal treatment temperature of the alumina films is set to $600{ }^{\circ} \mathrm{C}$ to ensure the majority amount of organics decomposed and the obtained alumina films is still in amorphous state, which meets our expectations [13]. Moreover, it can be concluded that the crystallization temperature of amorphous alumina is rising with the increasing of Si content. The possible reason is that $\mathrm{Si}$, as a network-forming cation, can stabilize the amorphous network structures which would improve the stability of alumina as well.

\subsection{Binding Structure and surface state}

In order to investigate the bond structure of Si-doped alumina thin film, FT-IR analysis was used. The selected region $\left(675 \mathrm{~cm}^{-1}-1600 \mathrm{~cm}^{-1}\right)$, which is related to the characteristic bonds of Si-doped alumina films, of the FT-IR absorption spectra is shown in Fig. 2. The region between $700 \mathrm{~cm}^{-1}$ and $1000 \mathrm{~cm}^{-1}$ is ascribed to Al-O stretch vibration [14]. Especially, vibration peaks near $900 \mathrm{~cm}^{-1}$ and $990 \mathrm{~cm}^{-1}$ may be attributed to Al-O-Al and Al-O-Si vibrations, respectively [15], indicating that Si ions participate in the construction of the structure of alumina film. The results meet the reason why the transforming temperature from amorphous $\mathrm{Al}_{2} \mathrm{O}_{3}$ to $\gamma-\mathrm{Al}_{2} \mathrm{O}_{3}$ rises with the increasing of Si concentration. Moreover, since little amount of organic matters are remained in the samples after the processing of annealing. Carbon bond vibrations such as $\mathrm{C}-\mathrm{C}\left(1430 \mathrm{~cm}^{-1}\right)$ and $\mathrm{C}=\mathrm{O}\left(1510 \mathrm{~cm}^{-1}\right)$ appeared in the $1200 \mathrm{~cm}^{-1}-1800 \mathrm{~cm}^{-1}$ region of the spectra.

XPS was employed to further explore the chemical binding of silicon in thin films. Fig. 3 shows the XPS data of alumina thin films. The binding energy is calibrated by locating the $\mathrm{C} 1 \mathrm{~s}$ level at $285.0 \mathrm{eV}$. As shown in figure 3 (a), Si element can be found from the XPS survey spectra of the Si-doped samples. Fig. 3 (b) displays 
the comparative Si $2 p$ core-level of thin films; the binding energy of $\mathrm{Si} 2 \mathrm{p}$ is $101.7 \mathrm{eV}$, $101.9 \mathrm{eV}$ and $102.1 \mathrm{eV}$ respectively, which corresponds to the $\mathrm{Si}-\mathrm{O}$ bond [16]. It is obvious that the peak shifts somewhat to higher binding energy and the counts of $\mathrm{Si}$ $2 \mathrm{p}$ increase with the increasing of Si concentration. It reveals that the more $\mathrm{Si}$ ions are added, the more Si-O bond will be formed. To identify specific types of Si-O bond, the selected high resolution spectrum of $\mathrm{O}$ 1s of sample $\mathrm{Si}-10 \%$ was investigated. The fitted component peaks are presented in Fig.4. It can be seen from the spectrum that $\mathrm{O}$ 1s has three peak positions, indicating that there are three different oxygen species presenting on the surface. The peaks at binding energy of $531.04 \mathrm{eV}, 531.70 \mathrm{eV}$ and $532.49 \mathrm{eV}$ are attributed to Al-O-Al, Al-O-Si and $\mathrm{H}_{2} \mathrm{O}$, respectively [17]. So it can be concluded that the existence form of silicon in alumina film is Al-O-Si, which is in accordance with the FT-IR result.

Fig. 5 is the surface morphology of $\mathrm{Si}-0 \%, \mathrm{Si}-2 \%, \mathrm{Si}-5 \%, \mathrm{Si}-10 \%$. There are no apparent differences in the surface morphology with the increasing of $\mathrm{Si}$ concentration. The surfaces are smooth and uniform which suggests that no crystallization of the undoped and Si-doped alumina happened after annealed at 600 ${ }^{\circ} \mathrm{C}$ for $3 \mathrm{~h}$. According to the above results, both phase structure and surface morphology are not affected by the Si doping. The thickness of the thin films measured by an interferometer was about $210 \mathrm{~nm}$.

\subsection{Dielectric properties}

Fig. 6 is the frequency dependence of dielectric constant and dielectric loss tangent of the $\mathrm{Al}_{2-\mathrm{x}} \mathrm{Si}_{\mathrm{x}} \mathrm{O}_{\mathrm{y}}$ thin films in the frequency range from $100 \mathrm{~Hz}$ to $2 \mathrm{MHz}$ at room temperature. The dielectric constant of the silicon doped amorphous alumina films is in the range of 7.52-8.53 at $100 \mathrm{KHz}$, which is just a little lower than the crystalline films. The dielectric loss tangent of the films is about 0.02-0.04 at 100 KHz. The overall dielectric spectra are typical characteristics of conduction dominant low loss linear dielectrics. No apparent dielectric relaxation at room temperature can be observed at low frequency range. Both dielectric constant and loss tangent decrease with the increase of frequency. It can be seen from Fig. 6 (a), the dielectric 
constant of Si-doped thin alumina films is lower than that of the undoped film and increases slightly with the increasing of Si doping concentration. From Fig. 6 (b), the dielectric loss tangent is strongly affected by the Si doping concentration. It should be noted, that the sample $\mathrm{Si}-2 \%$ exhibits much lower dielectric loss in the frequency range from $100 \mathrm{~Hz}$ to $100 \mathrm{KHz}$, The loss tangent of the sample $\mathrm{Si}-2 \%$ is only 0.019 at $100 \mathrm{~Hz}$, In contrast, the loss tangent for samples $\mathrm{Si}-5 \%$, Si-10\%, and $\mathrm{Si}-0 \%$ are 0.19 , 0.22 and 0.29 , respectively.

The high field dielectric behavior and breakdown phenomena of the Si doped amorphous alumina films are the major concern of this work. Fig. 7 is the current-voltage (I-V) characteristics of the silicon doped amorphous alumina films with $\mathrm{Au}$ top electrode covering a wide voltage/electric field range up to their breakdown voltage. As shown in Fig. 7, in the low voltage regime, the leakage current increases with the increasing of voltage in a non-ohmic way. The Si-doped alumina films exhibit lower leakage current in comparison with the undoped one. Silicon doping is able to reduce the leakage current of the alumina films. The leakage current of $\mathrm{Si}-2 \%$ sample is almost two orders of magnitude lower than that of the undoped one Si-0\%. With the increasing of silicon doping concentration up to $\mathrm{Si}-5 \%$ and $\mathrm{Si}-10 \%$, the leakage current increased but still lower than the undoped one, which is consistent with the dielectric loss tangent measurement exhibited in Fig. 6.

At medium and high voltage regime, the escalation of leakage current with increasing applied voltage slowed down, saturated and even reduced. During the I-V measurement, a lot of current spikes can be observed on the I-V curve, which corresponds to the recoverable soft breakdowns of the alumina films. Hundreds even thousands of soft breakdown events took place during the I-V measurement, which damaged the gold film electrode and resulted in the decrease of electrode area as shown in Fig. 8. The decrease of electrode area is bound to increase its resistivity and decrease of its leakage current.

Fig. 9 is the result of the nominal breakdown strength of the alumina films. The nominal breakdown strength of the Si-doped alumina films is much higher than that 
of the undoped alumina film (293 MV/m). However, breakdown strength of Si-doped alumina thin film decreased from $566 \mathrm{MV} / \mathrm{m}$ to $417 \mathrm{MV} / \mathrm{m}$ with the increase of $\mathrm{Si}$ concentration from $\mathrm{Si}-2 \%$ to $\mathrm{Si}-10 \%$. It can be concluded that doping with $\mathrm{Si}$ is able to dramatically reduce the leakage current and enhance the breakdown strength of the amorphous alumina films. For the $\mathrm{Si}-2 \%$ sample, its leakage current reduced two orders of magnitude and its breakdown strength increased by $93 \%$ in comparison with the undoped alumina film.

The silicon doping effects on its dielectric behavior of the amorphous alumina film can be well understood from defect chemistry. According to the defect chemistry, silicon doping in alumina film can be described as follows:

$$
3 \mathrm{SiO}_{2} \stackrel{2 \mathrm{Al}_{2} \mathrm{O}_{3}}{\longrightarrow} 3 \mathrm{Si}_{\mathrm{Al}}^{\cdot}+6 \mathrm{O}_{\mathrm{O}}+V_{\mathrm{Al}}^{\prime \prime \prime}
$$

According to the above defect chemistry reaction, three units of $\mathrm{SiO}_{2}$ replaces two units of $\mathrm{Al}_{2} \mathrm{O}_{3}$. Three $\mathrm{Si}^{4+}$ cations occupied three $\mathrm{Al}^{3+}$ cation sites creating positively charged impurity center and leave one cation site vacant. To keep charge balance the vacant cation site is negatively charged. It is well know that the electronegativity of $\mathrm{Si}$ is 1.8 which is slightly higher than 1.5 of Al. So Si-O bond is stronger than that of $\mathrm{Al}-\mathrm{O}$. Meanwhile, ionic radius of $\mathrm{Si}^{4+}$ is $0.039 \mathrm{~nm}$ smaller than that of $\mathrm{Al}^{3+}$, which is $0.057 \mathrm{~nm}$. Therefore, by means of silicon doping, a more stable and tighter structure can be formed, which much suppressed the intrinsic lattice deformation and relaxation polarization as well as the transportation characteristics of the dielectric medium resulting in apparent reduction of dielectric constant, dielectric loss tangent and leakage current of the Si-doped alumina film as observed in the previous measurements.

On the other hand, the newly created cation vacancies promoted the ionic mobility of the dielectric medium. Appropriate ionic species can be transported by the applied electric field toward the original defect region of the film to repair its structure imperfection. In general, the structure defect region has been stimulated to a higher local energy level above the normal average. There is a trend to restore back to its 
normal structure and average energy level as long as the transformation barrier for the structure restoring can be overcome. The combination of higher mobility of ionic species of the silicon doped alumina medium and higher applied electric field seems to be effective in this case rendering the silicon doped alumina film self-healing behavior and significantly enhances its breakdown strength. Combining the above two aspects together, more stable thin films with self-healing behavior can be obtained.

However, as silicon doping concentration exceeds an optimum limit, too much cation vacancies would jeopardize the structure stability of the dielectric medium under high applied electric field leading to the increase of leakage current and dielectric loss, degradation of breakdown strength. Sample Si-2\% exhibits the most excellent dielectric properties, whose leakage current reduced two orders of magnitude, dielectric loss significantly reduced with stable dielectric constant, its breakdown strength increased by $93 \%$, compared with undoped alumina film.

\section{Conclusions}

Dense and uniform amorphous alumina film can be prepared via sol-gel and spin coating route. Silicon doping is able to effectively reduce the leakage current and dielectric loss of the film and significantly enhance its breakdown strength. The optimum silicon doping concentration is about 2 mole $\%$. The leakage current of the $\mathrm{Si}-2 \%$ film can be effectively reduced for two orders of magnitude. The dielectric loss of the film at low frequencies can also be greatly suppressed, while the breakdown strength of the silicon doped alumina film can be increased by $93 \%$. FT-IR and XPS results revealed that silicon can be incorporated into the amorphous aluminum structure occupying Al lattice site forming Al-O-Si bonds and resulting in a stable and tighter structure to enhance its dielectric properties. Cation vacancies associated with silicon doping promoted the ionic mobility of the amorphous alumina, which would benefit to the self-repairing of the defects under electric field and increase the breakdown strength of the amorphous alumina films. However, with higher silicon doping concentration, the cation vacancy concentration increased, the amorphous alumina structure would become unstable, which degraded the dielectric behavior of 
the film again. It can be concluded that $\mathrm{Si}^{4+}$ is a feasible substitution candidate for alumina thin film to improve its dielectric properties. The Si-doped alumina thin film is a promising candidate for application in energy-storage capacitors in the future.

\section{Acknowledgments}

This work is supported by the Ministry of Science and Technology of China through 973-project (Grant Number 2015CB654601), National Science Foundation of China (Grant Number 51272177) and International Science \& Technology Cooperation Program of China (Grant Number 2013DFR50470).

\section{References}

[1] C. Liu, F. Li, L.P. Ma, H.M. Cheng, Advanced materials for energy storage, Advanced Materials, 22 (2010) 28-62.

[2] X. Zhang, Y. Shen, Q. Zhang, L. Gu, Y. Hu, J. Du, Y. Lin, C.W. Nan, Ultrahigh energy density of polymer nanocomposites containing $\mathrm{BaTiO}_{3} @ \mathrm{TiO}_{2}$ nanofibers by atomic-scale interface engineering, Advanced Materials, 27 (2015) 819-824.

[3] J. Li, L. Zhang, S. Ducharme, Electric energy density of dielectric nanocomposites, Applied Physics Letters, 90 (2007) 13290 (1-4).

[4] Q. Chen, Y. Shen, S. Zhang, Q.M. Zhang, Polymer-Based Dielectrics with High Energy Storage Density, Annual Review of Materials Research, 45 (2015) 433-458.

[5] N. Guo, S.A. DiBenedetto, P. Tewari, M.T. Lanagan, M.A. Ratner, T.J. Marks, Nanoparticle, Size, Shape, and Interfacial Effects on Leakage Current Density, Permittivity, and Breakdown Strength of Metal Oxide-Polyolefin Nanocomposites: Experiment and Theory, Chemistry of Materials, 22 (2010) 1567-1578.

[6] J. Lee, J. Kim, T. Hyeon, Recent Progress in the Synthesis of Porous Carbon Materials, Advanced Materials, 18 (2006) 2073-2094.

[7] M. Tsai, P. Chen, J. Do, Preparation and characterization of $\mathrm{Ppy} / \mathrm{Al}_{2} \mathrm{O}_{3} / \mathrm{Al}$ used as a solid-state capacitor, Journal of Power Sources, 133 (2004) 302-311.

[8] S. Jones, Sol-gel synthesis of magnesium silicate thin films and powders and synthesis and characterization of nickel-alumina composites, Michigan, 1995.

[9] M. Yao, R. Xiao, Y. Peng, J. Chen, B. Hu, X. Yao, The influence of titanium 
doping on the electric properties of amorphous alumina films prepared by sol-gel technology, Journal of Sol-Gel Science and Technology, 74 (2014) 39-44.

[10] P. Zou, M. Yao, J. Chen, Y. Peng, X. Yao, Leakage current and dielectric breakdown in lanthanum doped amorphous aluminum oxide films prepared by sol-gel, Ceramics International, 42 (2016) 4120-4125.

[11] X. Wang, Y. Guo, G. Lu, L. Jiang, Y. Guo, Z. Zhang, An excellent support of Pd catalyst for methane combustion: thermal-stable Si-doped alumina, Catalysis Today, 126 (2007) 369-374.

[12] O. Mekasuwandumrong, P. Tantichuwetrials, C. Chaisuk, P. Praserthdam, Impact of concentration and Si doping on the properties and phase transformation behavior of nanocrystalline alumina prepared via solvothermal synthesis, Materials Chemistry and Physics, 107 (2008) 208-214.

[13] W. Shan, M. Yao, B. Hu, P. Yang, X. Yao, Preparation and electrical propertoes of aluminum oxide thin films by sol-gel process, Functional Materials, 44 (2013) $581-584$.

[14] Verlaan, V.van den Elzen, L. R. J. G.Dingemans, G.van de Sanden, M. C.

M.Kessels, W. M. M. Composition and bonding structure of plasma-assisted ALD $\mathrm{Al}_{2} \mathrm{O}_{3}$ films, Physica Status Solidi (c),3-4 (2010) 976-979.

[15] L. Stoch, M. Sroda, Infrared spectroscopy in the investigation of oxide glasses structure, journal of molecular structure, 511-512 (1999) 77-84.

[16] M. Todea, B. Frentiu, R.F.V. Turcu, P. Berce, S. Simon, Surface structure changes on aluminosilicate microspheres at the interface with simulated body fluid, Corrosion Science, 54 (2012) 299-306.

[17] NIST X-ray Photoelectron Spectroscopy Database. http://srdata.nist.gov/xps/, 2012. 


\section{Figure captions:}

Fig. 1. DSC curves of $\mathrm{Si}-0 \%, \mathrm{Si}-2 \%, \mathrm{Si}-5 \%$ and $\mathrm{Si}-10 \%$ at $10{ }^{\circ} \mathrm{C} / \mathrm{min}$

Fig. 2. FT-IR spectra of $\mathrm{Al}_{2-\mathrm{x}} \mathrm{Si}_{\mathrm{x}} \mathrm{O}_{\mathrm{y}}$ thin films with $0,2,5,10 \mathrm{~mol} \% \mathrm{Si}$ doping

Fig. 3. (a) the survey spectra of alumina films with $\mathrm{Si}-0 \%$, $\mathrm{Si}-2 \%, \mathrm{Si}-5 \%, \mathrm{Si}-10 \%$. (b) shows the comparative high resolution spectrum of Si $2 p$

Fig. 4. XPS spectrum of O 1s for Si-10\% thin film

Fig. 5. Surface morphologies of $\mathrm{Al}_{2-\mathrm{x}} \mathrm{Si}_{\mathrm{x}} \mathrm{O}_{\mathrm{y}}$ thin films, (a) $\mathrm{Si}-0 \%$,(b) $\mathrm{Si}-2 \%$, (c) $\mathrm{Si}-5 \%$, (d) $\mathrm{Si}-10 \%$

Fig. 6. The $\mathrm{V} v s$. I plots for the $\mathrm{Al}_{2-\mathrm{x}} \mathrm{Si}_{\mathrm{x}} \mathrm{O}_{\mathrm{y}}$ thin films

Fig. 7. SEM image of the surface of Au electrode after breakdown. Inset is an enlarged diagram of a breakdown plot

Fig. 8. Weibull plots of breakdown voltage of $\mathrm{Al}_{2-\mathrm{x}} \mathrm{Si}_{\mathrm{x}} \mathrm{O}_{\mathrm{y}}$ films. Inset dielectric strength of the samples ( $U$ is the specific breakdown voltage of each specimen, $P_{i}$ is the cumulative failure probability and $\mathrm{E}$ is the average breakdown voltage)

Fig. 9. Frequency dependence of (a) dielectric constant, (b) dielectric loss of the $\mathrm{Al}_{2-\mathrm{x}} \mathrm{Si}_{\mathrm{x}} \mathrm{O}_{\mathrm{y}}$ thin films. 
Fig. 1. DSC curves of $\mathrm{Si}-0 \%, \mathrm{Si}-2 \%, \mathrm{Si}-5 \%$ and $\mathrm{Si}-10 \%$ at $10{ }^{\circ} \mathrm{C} / \mathrm{min}$

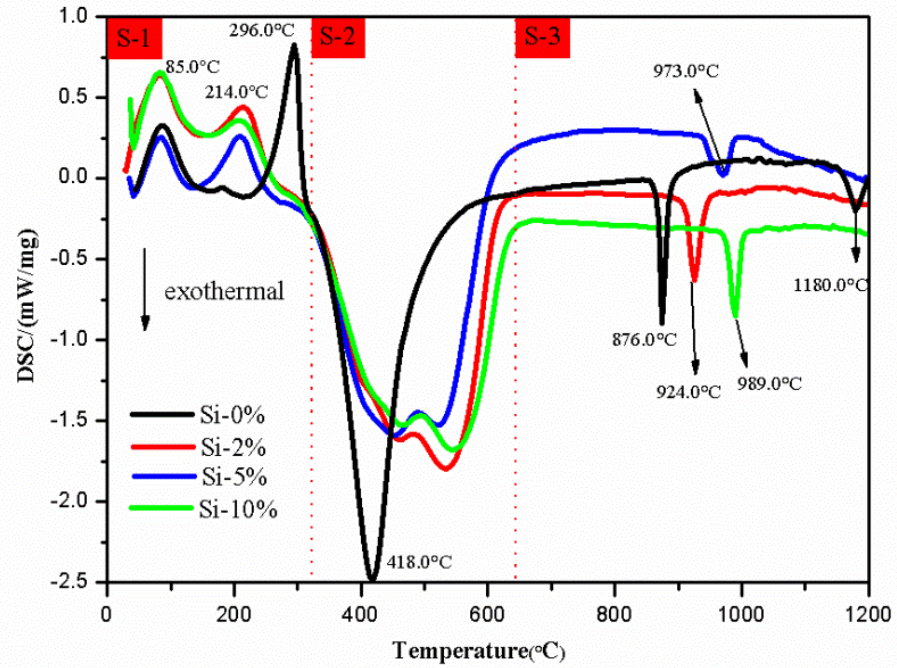


Fig. 2. FT-IR spectra of $\mathrm{Al}_{2-\mathrm{x}} \mathrm{Si}_{\mathrm{x}} \mathrm{O}_{\mathrm{y}}$ thin films with $0,2,5,10 \mathrm{~mol} \%$ Si doping

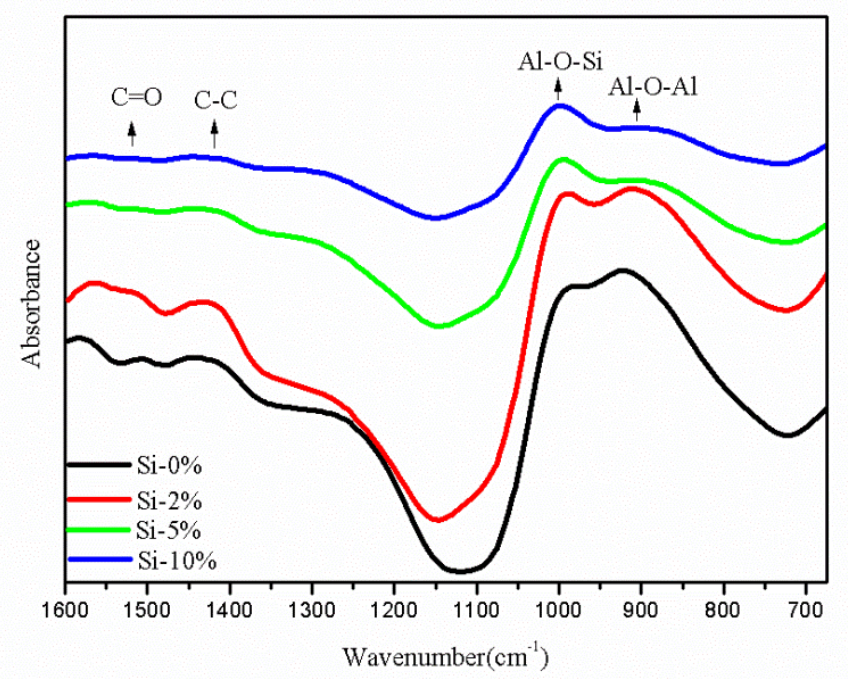


Fig. 3. (a) the survey spectra of alumina films with $\mathrm{Si}-0 \%$, $\mathrm{Si}-2 \%, \mathrm{Si}-5 \%, \mathrm{Si}-10 \%$. (b) shows the comparative high resolution spectrum of $\mathrm{Si} 2 \mathrm{p}$
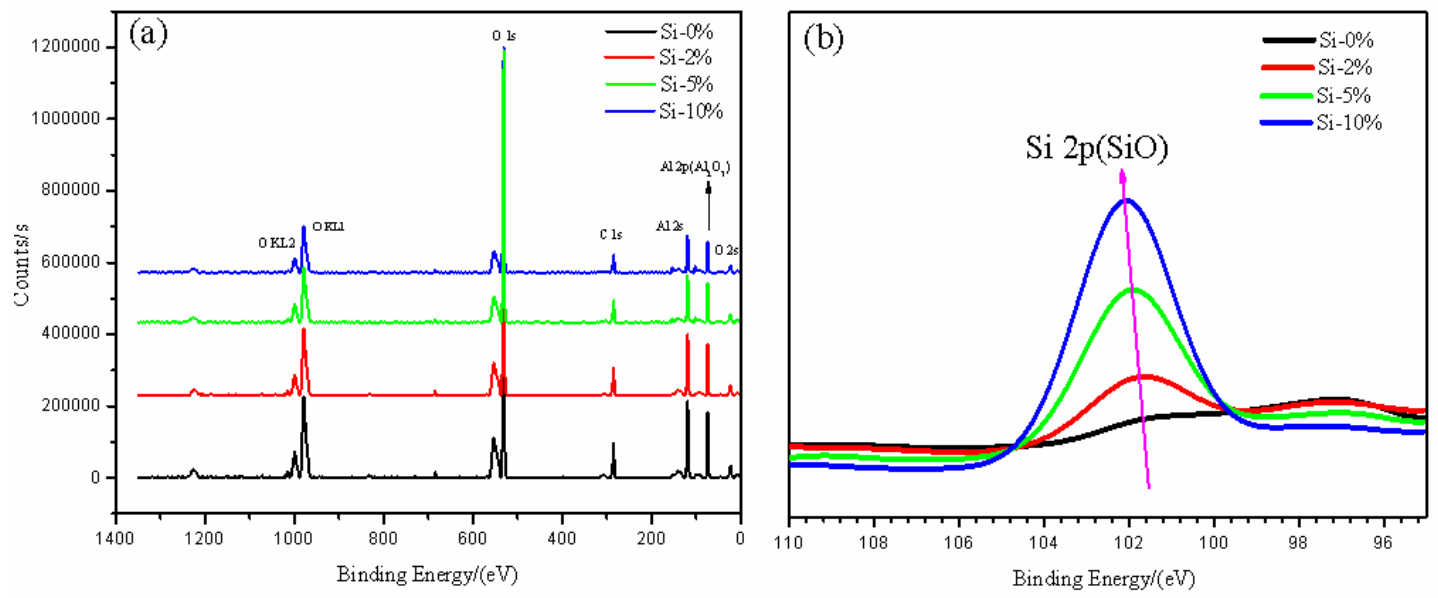
Fig. 4. XPS spectrum of O 1s for Si-10\% thin film

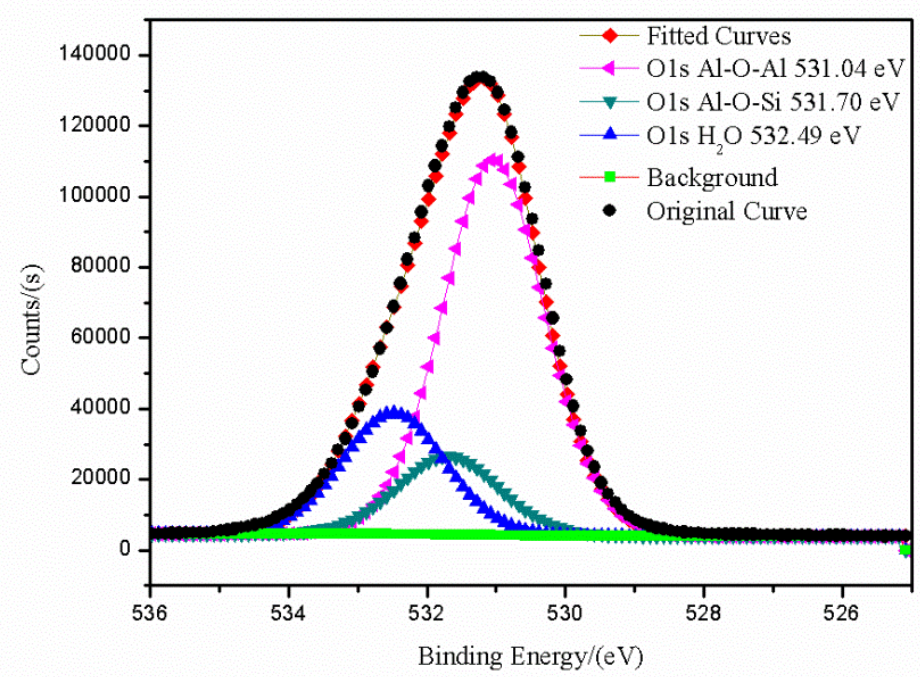


Fig. 5. Surface morphologies of $\mathrm{Al}_{2-\mathrm{x}} \mathrm{Si}_{\mathrm{x}} \mathrm{O}_{\mathrm{y}}$ thin films, (a) $\mathrm{Si}-0 \%$, (b) $\mathrm{Si}-2 \%$, (c) $\mathrm{Si}-5 \%$, (d) $\mathrm{Si}-10 \%$

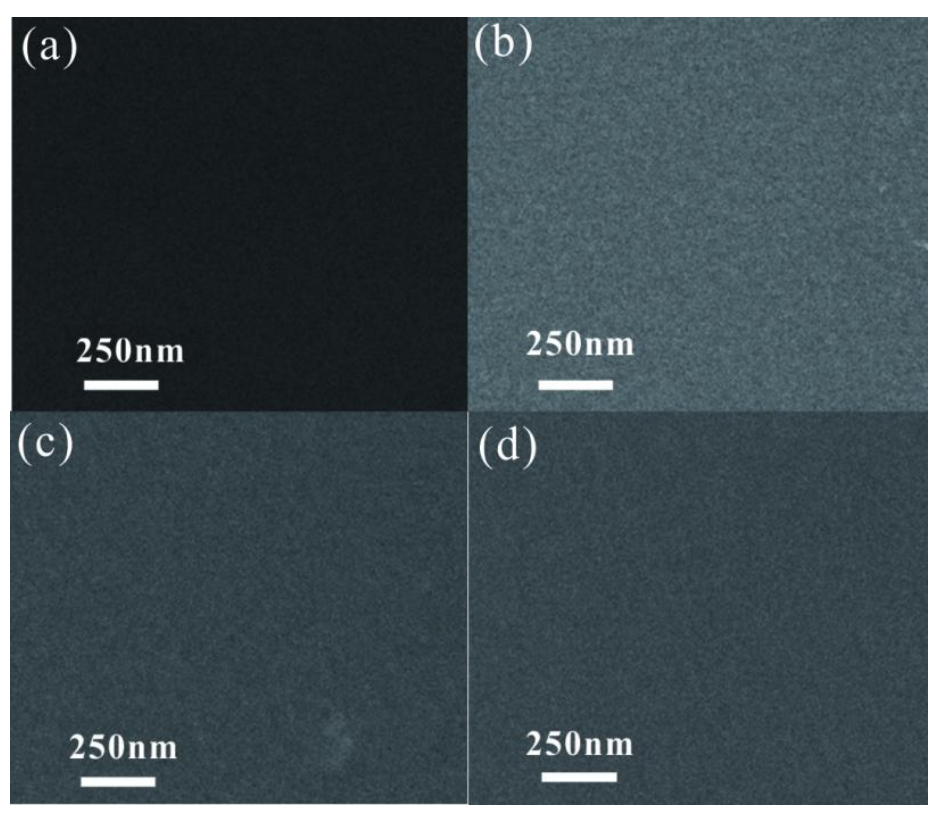


Fig. 6. Frequency dependence of (a) dielectric constant, (b) dielectric loss of the $\mathrm{Al}_{2-\mathrm{x}} \mathrm{Si}_{\mathrm{x}} \mathrm{O}_{\mathrm{y}}$ thin films
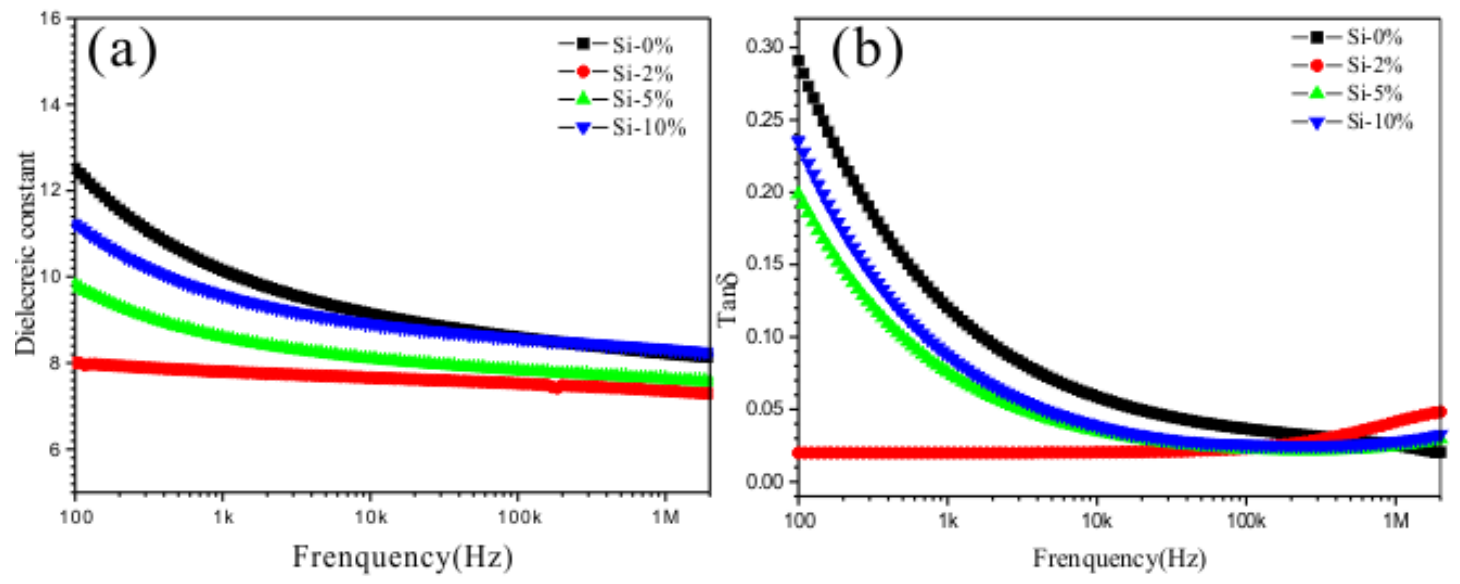
Fig. 7. The $\mathrm{V} v s$. I plots for the $\mathrm{Al}_{2-\mathrm{x}} \mathrm{Si}_{\mathrm{x}} \mathrm{O}_{\mathrm{y}}$ thin films

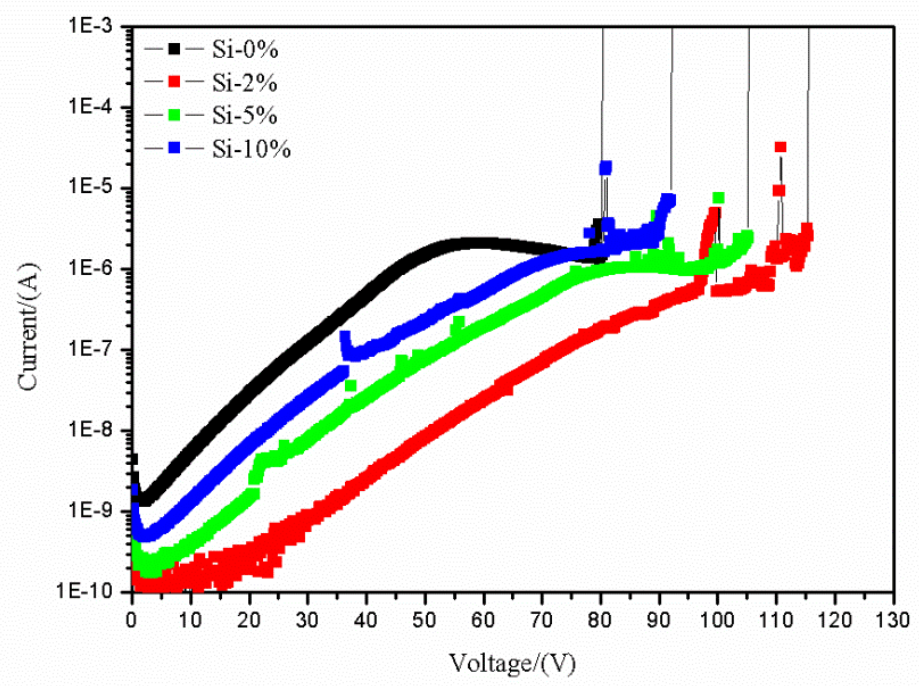


Fig.8 SEM image of the surface of Au electrode after breakdown. Inset is an enlarged diagram of a breakdown plot

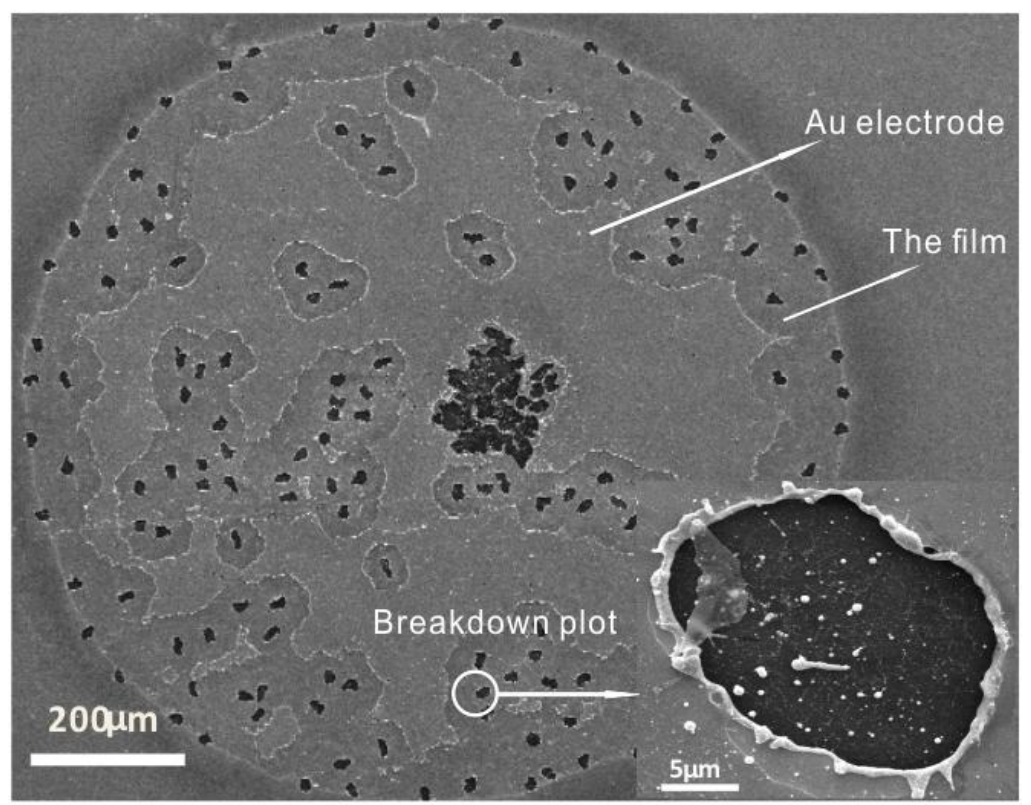


Fig. 9. Weibull plots of breakdown voltage of $\mathrm{Al}_{2-\mathrm{x}} \mathrm{Si}_{\mathrm{x}} \mathrm{O}_{\mathrm{y}}$ films. Inset dielectric strength of the samples ( $U$ is the specific breakdown voltage of each specimen, $P_{i}$ is the cumulative failure probability and $\mathrm{E}$ is the average breakdown voltage)

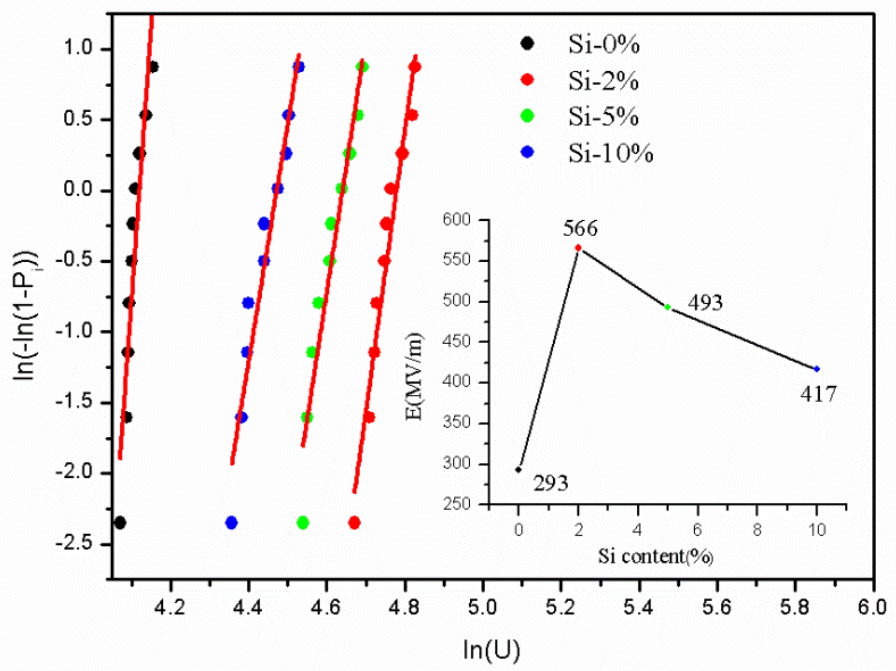

